\title{
Über die Methodik der Probenahme zur Untersuchung von Abwasserreinigungsanlagen
}

\author{
von F. ZEHENDER, Zürich
}

Mitteilung aus der Eidg. Anstalt für Wasserversorgung, Abwasserreinigung und Gewässerschutz an der Eidgenössischen Technischen Hochschule Zürich

\section{Einleitung}

In den Lehrbüchern der Abwasseruntersuchung wird der Entnahmemethode von Wasserproben stets große Bedeutung beigemessen. Dies ist namentlich dann begründet, wenn beispielsweise aus den Analysenergebnissen auf die gesamte Tagesmenge eines Stoffes geschlossen oder wenn die Reinigungswirkung einer Anlage ermittelt werden soll. Um bei der Untersuchung sachgemäß vorgehen zu können, sind hauptsächlich folgende Umstände zu berücksichtigen.

1. Das zu untersuchende Abwasser ist einem ständigen Wechsel unterworfen, indem seine Menge und ungefähr gleichzeitig sein Gehalt an Schmutzstoffen mit einer gewissen GesetzmäBigkeit in einem täglich sich wiederholenden, einigermaßen wellenförmigen Verlauf zu- und abnehmen. Infolge von Witterungseinflüssen und aus anderen Gründen können jedoch an einzelnen Tagen deutliche Abweichungen vom normalen Verhalten eintreten.

2. Eine Abwasserreinigungsanlage arbeitet im Durchflußbetrieb, wird also ununterbrochen vom zu reinigenden Wasser durchflossen, vergleiche IMHOFF (1), Die erwähnten täglich wiederkehrenden Konzentrationsschwankungen sind bis zu einem gewissen Grade auch in der Anlage anzutreffen. In welchem Maße dies jeweilen der Fall ist, hängt von der Bauweise und den Betriebsbedingungen der Anlage ab. Unterschiede in der Wassermenge wirken sich auf die Aufenthaltszeit des Wassers in der Anlage aus.

3. Im Abwasser liegen oft leicht zersetzliche, labile Stoffe vor. Ferner bewirken Bakterien einen rasch einsetzenden Abbau bestimmter Substanzen. Infolgedessen sollten die chemischen Analysen des Wassers sofort durchgeführt werden.

Die angedeuteten, für die Probenahme wesentlichen Bedingungen sind von verschiedenen Autoren eingehend besprochen und bei der Aufstellung entsprechender Vorschriften berücksichtigt worden, BACH (2), MEINCK (3), 
BABBITT (4), THEROUX, ELDRIDGE und MALLMANN (5). Aus ihren Angaben lassen sich kurz die wichtigsten Grundsätze, welche bei der Probenahme zu befolgen sind, zusammenfassen. Es werden sog. Einzel- und Sammelproben unterschieden. Da die Zusammensetzung des Abwassers ständig wechselt, sagt die Untersuchung einer Einzelprobe nicht sehr viel. Dagegen geben Sammelproben den durchschnittlichen Gehalt eines Stoffes während eines bestimmten Zeitraums an, welche Größe den Verunreinigungsgrad eines Gewässers zuverlässiger anzugeben vermag. Als Zeiteinheit bei Abwasseruntersuchungen gilt in der Regel der ganze Tag (24 Stunden), da sich der periodische Wechsel hinsichtlich Menge und Konzentration einmal täglich vollzieht. Eine in regelmäßigen Zeitabständen erhobene Reihe von Einzelproben läßt den zeitlichen Verlauf in der Zusammensetzung des Wassers erkennen, BACH (2). Wegen der leichten Zersetzlichkeit des Abwassers besteht die Tendenz, die Wartezeit zwischen der Probenahme und der Analyse möglichst abzukürzen. BACH (2) schreibt deshalb vor, Sammelproben während längstens sechs Stunden zu entnehmen und hierauf sofort zu untersuchen.

Bei der Untersuchung von Reinigungsanlagen, wobei die Reinigungswirkung in Zahlenwerten erfaßt werden soll, sind grundsätzlich die Ergebnisse der Proben des Zuflusses und des Abflusses einander gegenüberzustellen. Doch ist darauf $z u$ achten, daß einander entsprechende Proben verglichen werden. Demgemäß ist die Zeit zu berücksichtigen, welche das Wasser zum Durchfließen der Anlage benötigt, das heißt es sind sogenannte korrespondierende Proben zu entnehmen, TILLMANS (7), MEINCK (3). In der Literatur finden sich jedoch keine Angaben, ob den korrespondierenden Einzel- oder Sammelproben der Vorzug zu geben ist, bzw. ob der Grundsatz, korrespondierende Proben untereinander $\mathrm{zu}$ vergleichen, für die verschiedenen Probenahmemethoden in jedem Falle genügt. Strenge genommen darf ein Vergleich von Proben des Zu- und Abflusses nur dann erfolgen, wenn ein bestimmtes Wasservolumen beim Durchfließen der Anlage sich nicht mit anderem Wasser vermischt. Dies ist jedoch in Reinigungsanlagen niemals der Fall. Infolge ihrer hydraulischen Eigenschaften und den jeweils herrschenden Betriebsbedingungen finden stets bis $\mathrm{zu}$ einem gewissen Grade Vermischungen des in seiner Konzentration wechselnden, durch die Anlage fließenden Wassers statt, so daß bei strenger Betrachtung ein direkter Vergleich von korrespondierenden Proben nur unter ganz bestimmten Bedingungen zulässig ist. Es bleibt jedoch zu prüfen, in welchem Maße sich diese Erscheinungen bei gegebenen Verhältnissen in einer Reinigungsanlage auswirken und inwiefern diese Umstände bei der Entnahme von Proben von Bedeutung sind.

In der vorliegenden Abhandlung berichten wir über Versuche, in wel- 
chen die hydraulischen Eigenschaften von biologischen Reinigungsanlagen systematisch untersucht wurden. Die verschiedenen Probenahme- und Untersuchungsmethoden zur Ermittlung der Reinigungswirkung von Anlagen oder von Verfahren werden diskutiert und auf Grund der erhaltenen Ergebnisse hinsichtlich ihrer Zuverlässigkeit geprüft.

\section{Die Methoden zur quantitativen Ermittlung der Reinigungswirkung von Abwasserreinigungsanlagen}

Um in den nachstehenden Ausführungen die bei den verschiedenen Fragestellungen verwendeten Begriffe klar auseinanderhalten zu können, seien zunächst die im einzelnen zu ermittelnden Größen zusammengestellt. Es wird grundsätzlich zwischen dem Gehalt eines Stoffes im Abwasser und der in einem bestimmten Zeitabschnitt durchgeflossenen gesamten Stoffmenge unterschieden. Demgemäß werden die nachstehend aufgeführten Werte bestimmt, wobei als Zeiteinheit für die Sammelproben stets 24 Stunden gewählt sind.

1. Der Gehalt eines Stoffes in $\mathrm{mg} / \mathrm{l}$

a) in einem bestimmten Zeitpunkt,

b) bei mehrmaliger Bestimmung in Abhängigkeit der Zeit,

c) als Durchschnittswert von 24 Stunden.

2. Die Gesamtmenge eines Stoffes, welche während eines ganzen Tages durchfließt, angegeben in $\mathrm{kg} / \mathrm{Tag}$.

Werden die Werte auf Grund von Sammelproben («Mischproben», "composite samples") bestimmt, so ist nach den Vorschriften der Literatur vorzugehen, «STANDARD METHODS» (6), TILLMANS (7), "EINHEITSVERFAHREN" (8). Zur Bestimmung des durchschnittlichen Gehaltes eines Stoffes am Versuchstage werden gleiche Volumina der einzelnen Wasserproben entnommen und zur Sammelprobe gemischt. Soll jedoch die gesamte Stoff menge des Tages ermittelt werden und wechselt die Wassermenge im Laufe eines Tages, so ist das Volumen der einzelnen Proben, welche zur Sammelprobe vereinigt werden, proportional der im betreffenden Zeitpunkt fließenden Menge zu wählen.

Die Reinigungswirkung von Anlagen wird entsprechend den oben festgelegten Werten angegeben als

1. Abnahme des durchschnittlichen Tagesgehaltes eines Stoffes oder einer Kennzahl in mg/l oder in Prozenten. Diese Größe wird als Reinigungseffekt bezeichnet. Ihre Ermittlung erfolgt ohne Berücksichtigung der im Tag abgebauten Gesamtmenge des Stoffes.

2. Abnahme der Gesamttagesmenge eines Stoffes oder einer Kennzahl in $\mathrm{kg} / \mathrm{Tag}$ oder in Prozenten. Aus diesem Wert ergibt sich der 
Gesamttagesumsatz. Er dient zur Berechnung der Leistung einer Anlage, welche unter Berücksichtigung der Größe des Belüftungsraumes erfolgt und bildet allgemein die Grundlage für Bilanzuntersuchungen.

Um die für einen Versuchstag maßgebliche Reinigungswirkung einer Anlage oder eines Verfahrens einwandfrei zu bestimmen, sind somit je vom Zufluß und vom Abfluß korrespondierende Sammelproben (hinsichtlich Gehalt oder Menge der Stoffe) während 24 Stunden zu untersuchen, wobei nach BACH (2) zudem eine Unterteilung in vier Sammelproben zu sechs Stunden vorgenommen werden soll.

Die erwähnten, sich über 24 Stunden erstreckenden Probenahmemethoden dienen zur bilanzmäßigen Leistungsprüfung einer Anlage. Da alle während eines Tages eintretenden Bedingungen, d. h. schwache und starke Belastungen einbezogen sind, kann den so erhaltenen Ergebnissen allgemeine Gültigkeit zugesprochen werden.

Neben solchen, verhältnismäßig umständlichen Untersuchungen werden aber auch vereinfachte Methoden angewendet, welche sich auf kürzere Zeitabschnitte beziehen. Es sind dies die regelmäßig durchzuführenden B et ri ebs kontrollen. Sie können lediglich in der Untersuchung des Abflusses bestehen, oder es wird auf Grund von Einzel- bzw. Sammelproben während einiger Stunden die Beziehung zwischen Zu- und Abfluß gesucht. Da es sehr wertvoll wäre, stets die gleichen, oben aufgeführten Werte für die Reinigungswirkung einer Anlage zu verwenden, sei hier angeregt, für die Betriebskontrollen solche vereinfachte Methoden zu wählen, welche wenigstens überschlagsmäßig zu den erwähnten Größen führen (Abnahme des durchschnittlichen Tagesgehaltes oder der Gesamttagesmenge). Dies ist möglich, wenn der tägliche Verlauf des Abwasserzuflusses (Menge und Konzentration) der betreffenden Anlage einigermaßen bekannt ist und wenn am Versuchstage keine Ausnahmebedingungen herrschen. Wie dies erreicht werden kann, soll noch an einem Beispiel gezeigt werden.

\section{Durchgeführte Untersuchungen}

Die nachstehend aufgeführten Versuche sind in der Versuchsanlage Werdhölzli der Eidg. Anstalt für Wasserversorgung, Abwasserreinigung und Gewässerschutz in Verbindung mit anderen Untersuchungen durchgeführt worden. Um für die Praxis gültige Ergebnisse zu erhalten, wurden die gestellten Fragen an normal betriebenen, biologischen Reinigungsanlagen technischen Maßstabs geprüft. Hinsichtlich Größe der untersuchten Anlagen und in bezug auf die Abwasserzusammensetzung lagen Verhältnisse vor, wie sie allgemein vorkommen können, indem geklärtes Abwasser der Stadt Zürich von rund 1500-3000 Einwohnern von einem Reinigungssystem verarbeitet wurde. Dagegen erfolgte die Beschickung über die ganze Versuchszeit mit konstanter Wassermenge, was somit einem gewöhnlich nicht vorkommenden Spezialfall entspricht. 
1. Die hydraulischen Eigenschaften von Reinigungsanlagen, welche mit konstanter Wassermenge beschickt werden

Eine Reihe von Faktoren, wie Größe und Form einer Anlage, herrschende Betriebsbedingungen, bestimmen die hydraulischen Eigenschaften eines Reinigungssystems. Sie sind maßgebend für den Durchfluß des Wassers durch die Anlage, das heißt für die Durchflußzeit, für allfällig eintretende Vermischungsvorgänge und für Konzentrationsänderungen. Die Untersuchung der hydraulischen Bedingungen der im Betriebe befindlichen Anlagen läßt sich somit mit Hilfe von Gehaltsbestimmungen durchführen, sofern dies Stoffe betrifft, welche durch die Reinigung nicht beeinflußt werden. Wir führten dies in einer Reihe von Versuchen in der Weise durch, daß wir jeweils den Gehalt des Chlorids bestimmten. Dabei stützten wir uns auf die Angabe von MEINCK (3), wonach das Chlorid des Abwassers in einer Reinigungsanlage eine durch die Reinigung «nicht berührte, unveränderliche Größe» darstelle. Wie nachstehend noch gezeigt werden wird, traf dies bei den von uns geprüften Reinigungsanlagen weitgehend zu. Gelegentlich festgestellte geringe Abweichungen vom normalen Verhalten vermögen unsere Schlußfolgerungen nicht $\mathrm{zu}$ beeinflussen.

Durch die systematisch durchgeführten Chloriduntersuchungen im Zu- und Abfluß einer Reinigungsanlage wird somit einerseits die Durchflußzeit bestimmt. Anderseits wird festgestellt, ob und in welchem Maße in korrespondierenden Einzel- oder Sammelproben Konzentrationsunterschiede vorliegen, um daraus auf die hydraulischen Verhältnisse der Anlage schließen zu können.

Versuchsbeispiel. Am Versuchstage wurden von zwei im Betriebe befindlichen Belebtschlammanlagen an drei Stellen, je beim Zufluß und beim Abfluß, in regelmäßigen Zeitabständen während 24 Stunden Proben entnommen und deren Chloridgehalt bestimmt. Einzelheiten über die verwende-

Tabelle 1

\begin{tabular}{|c|c|c|c|}
\hline & & Z-Verfahren & $\begin{array}{l}\text { Belebtschlemm- } \\
\text { verfahren }\end{array}$ \\
\hline Inhalt des Belebungsbeckens & $m^{3}$ & 77 & 193 \\
\hline Inhalt des Absetzbeckens . . & $\mathrm{m}^{3}$ & 72 & 72 \\
\hline Wassermenge (konstant) & $1 / \mathrm{sec}$ & 15 & 8 \\
\hline Menge des Rücklaufschlamms & $1 / \mathrm{sec}$ & 8 & 3 \\
\hline
\end{tabular}


ten, hinsichtlich Form und Anlageteile gleichen, hinsichtlich Größe der Belebungsbecken verschiedenen Anlagen finden sich bei KROPF (9). Die beiden parallel geschalteten Verfahren waren

a) das Zigerli-Verfahren, ein Teilreinigungsverfahren, ZIGERLI (10),

b) das Belebtschlammverfahren, betrieben als Vollreinigung.

Die Form derBelebungsbecken und dieBelüftungsart sind nach KESSENER (11) ausgebildet. Die wichtigsten Angaben über die Anlagen und die am Versuchstage maßgebenden Betriebsdaten sind in Tabelle 1 zusammengestellt.

Am gleichen Versuchstage wurden ferner mit Hilfe von automatisch arbeitenden Vorrichtungen während 24 Stunden korrespondierende Sammelproben erhoben, nämlich eine während 24 Stunden und vier einzelne während je sechs Stunden, vgl. Tabelle 3.

Die Versuchsergebnisse. Abbildung 1 zeigt den zeitlichen Verlauf der Chloridgehalte an den drei Probenahmestellen, je beim Zu- und Abfluß der beiden Anlagen. Die verhältnismäßig großen Konzentrationsschwankungen im Zufluß werden beim Durchfluß durch die Anlagen weitgehend ausgeglichen. Werden aus den drei Kurven je die korrespondierenden Punkte herausgegriffen, vergleicht man also korrespondierende Einzelproben, so gelangt man zu den in Tabelle 2 zusammen$m g \mathrm{Cl}^{\prime} / \mathrm{L}$

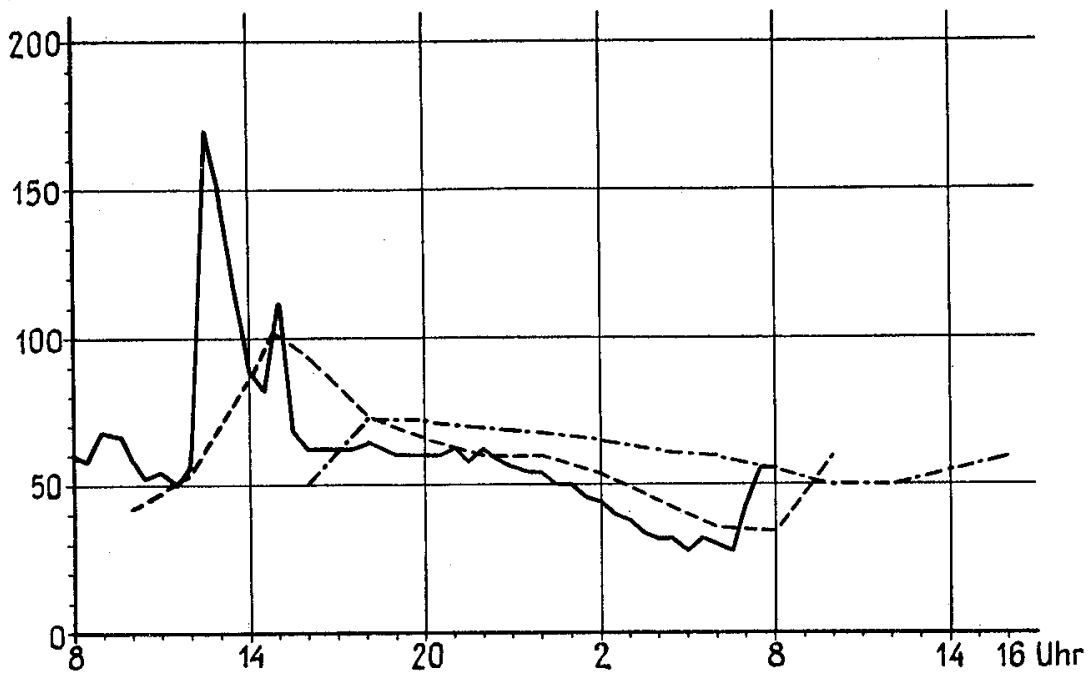

Abb. 1. Chloridgehalt im Zu- und Abfluß zweier Reinigungsanlagen am 22./23. 1. 47 (Z-Verfahren und Vollreinigung). Zufluß, - - - Abfluß Z-Verfahren, Abfluß Vollreinigung 
gestellten Werten, wobei je drei charakteristische Punkte (Maxima, Minima, Mittel) ausgewählt wurden.

Tabelle 2

\begin{tabular}{|c|c|c|c|c|c|c|}
\hline & \multicolumn{2}{|c|}{ Zufluß } & \multicolumn{2}{|c|}{ Abfluß Z } & \multicolumn{2}{|c|}{ Abfluß V } \\
\hline & Zeit & $\mathrm{mg} / \mathrm{L}$ & Zeit & $\mathrm{mg} / \mathrm{L}$ & Zeit & $\mathrm{mg} / \mathrm{L}$ \\
\hline Maximum & 12.30 & 170 & 15.00 & 102 & etwa 19.00 & 72 \\
\hline Minimum . & etwa 06.00 & 28 & etwa 08.00 & 34 & etwa 12.00 & 50 \\
\hline Mittel & 21.00 & 62 & etwa 23.30 & 60 & etwa 03.30 & 63 \\
\hline
\end{tabular}

Aus der Tabelle lassen sich die Durchflußzeiten der beiden Anlagen ablesen, was am besten auf Grund der ausgeprägten Maxima geschieht. Sie betragen etwa $2 \frac{1}{2}$ Stunden in der Z-Anlage und etwa $6 \frac{1}{2}$ Stunden in der Vollreinigungsanlage. Aus der Tabelle geht ferner hervor, daß in bestimmten Fällen deutliche Konzentrationsänderungen eintreten können. Ein Gehaltsmaximum im Zufluß wird in der Anlage herabgesetzt, ein Minimum dagegen heraufgesetzt. Ein mittlerer Gehalt im ZufluB wird jedoch nur wenig oder nicht geändert.

In Tabelle 3 und in Abbildung 2 sind die Chloridwerte von korrespondierenden Sammelproben zusammengestellt. Sie wurden teils direkt bestimmt, teils aus den Analysenresultaten der Einzel- oder Sammelproben berechnet. Die Tabelle zeigt, daß die in diesem Versuch angewandten, verschiedenen Arten der Probenahme bei einander entsprechenden Proben praktisch zum gleichen Ergebnis geführt haben. Aus dem Vergleich einzelner korrespondierender Sammelproben geht hervor:

a) 24-Stunden-Sammelproben weisen an allen Probenahmestellen gleichen Chloridgehalt auf.

b) 6-Stunden-Sammelproben verhalten sich ähnlich wie Einzelproben, indem bei ihnen die Tagesschwankungen deutlich zum Ausdruck kommen. Demgemäß erfährt das Rohwasser mit einem über dem Tagesdurchschnitt liegenden Chloridgehalt in der Anlage eine Konzentrationsabnahme. Liegt dagegen der Chloridgehalt des Rohwassers unter dem Tagesdurchschnitt, so erfolgt eine Zunahme.

c) Zwischen 8 und 20 Uhr entnommene 12-Stunden-Sammelproben, welche also die hohen Tageskonzentrationen enthalten, lassen auf eine 
Tabelle 3

Chloridgehalt $\left(\mathrm{mgCl}^{\prime} / 1\right)$ von korrespondierenden Sammelproben zu 6,12 und 24 Stunden

(Z-Verfahren und Vollreinigung, 22./23. 1.47)

\begin{tabular}{|c|c|c|c|c|c|c|c|c|}
\hline Probenahmestelle & \multicolumn{2}{|c|}{ Zufluß } & \multicolumn{3}{|c|}{ Abfluß "Z» } & \multicolumn{3}{|c|}{$A b f l u ß \ll V »$} \\
\hline Zeit & \begin{tabular}{|c|c|}
8 & 14 \\
bis & bis \\
14 & 20 \\
\end{tabular} & \begin{tabular}{|c|c}
20 & 2 \\
bis & bis \\
2 & 8 \\
\end{tabular} & & & & & & \\
\hline Nr. & \begin{tabular}{|l|l|}
1 & 2 \\
\end{tabular} & \begin{tabular}{l|l}
3 & 4
\end{tabular} & 1 & 23 & 4 & 1 & 23 & \begin{tabular}{l|l}
3 & 4
\end{tabular} \\
\hline $\begin{array}{l}\text { 6-Stunden-Mittel } \\
\text { aus Einzelproben berechnet } \\
\text { in Sammelproben gefunden. }\end{array}$ & \begin{tabular}{|l|l|}
81 & 69 \\
85 & 69 \\
\end{tabular} & \begin{tabular}{|l|l}
55 & 37 \\
58 & 34
\end{tabular} & & \begin{tabular}{l|l}
72 & 56 \\
73 & 56
\end{tabular} & \begin{tabular}{l|l}
6 & 41 \\
6 & 41
\end{tabular} & $\begin{array}{l}68 \\
66\end{array}$ & \begin{tabular}{l|l}
67 & 5 \\
69 & 5
\end{tabular} & \begin{tabular}{l|l}
57 & 53 \\
55 & 55
\end{tabular} \\
\hline Zeit & $8-20$ & $20-8$ & & & & & & \\
\hline Nr. & 1 & 2 & 1 & & 2 & 1 & & 2 \\
\hline $\begin{array}{l}\text { 12-Stunden-Mittel } \\
\text { aus Einzelproben berechnet. } \\
\text { aus Sammelproben berechnet. }\end{array}$ & $\begin{array}{l}75 \\
77\end{array}$ & $\begin{array}{l}46 \\
46\end{array}$ & $\begin{array}{l}71,5 \\
73\end{array}$ & & $\begin{array}{l}48,5 \\
48,5\end{array}$ & $\begin{array}{l}67, \\
67,\end{array}$ & & $\begin{array}{l}55 \\
55\end{array}$ \\
\hline Zeit & $14-2$ & $2-14$ & & & & & & \\
\hline $\mathrm{Nr}$. & 1 & 2 & 1 & & 2 & 1 & & 2 \\
\hline $\begin{array}{l}12 \text {-Stunden-Mittel } \\
\text { aus Einzelproben berechnet. } \\
\text { aus Sammelproben berechnet. }\end{array}$ & $\begin{array}{l}62 \\
63,5\end{array}$ & $\begin{array}{l}59 \\
59,5\end{array}$ & $\begin{array}{l}64 \\
64,5\end{array}$ & & $\begin{array}{l}56 \\
57\end{array}$ & $\begin{array}{l}62 \\
62\end{array}$ & & $\begin{array}{l}60,5 \\
60,5\end{array}$ \\
\hline Zeit & & -8 & & & & & & \\
\hline Nr. & & 1 & & 1 & & & 1 & \\
\hline $\begin{array}{l}\text { 24-Stunden-Mittel } \\
\text { aus Einzelproben berechnet } \\
\text { aus } 6 \text {-Std.-Sammelpr. berechn. } \\
\text { in 24-Std.-Sammelpr.gefunden }\end{array}$ & & $\begin{array}{l}0,5 \\
1,5 \\
1\end{array}$ & & $\begin{array}{l}60 \\
61,5 \\
59\end{array}$ & & & $\begin{array}{l}61 \\
61 \\
57\end{array}$ & \\
\hline
\end{tabular}



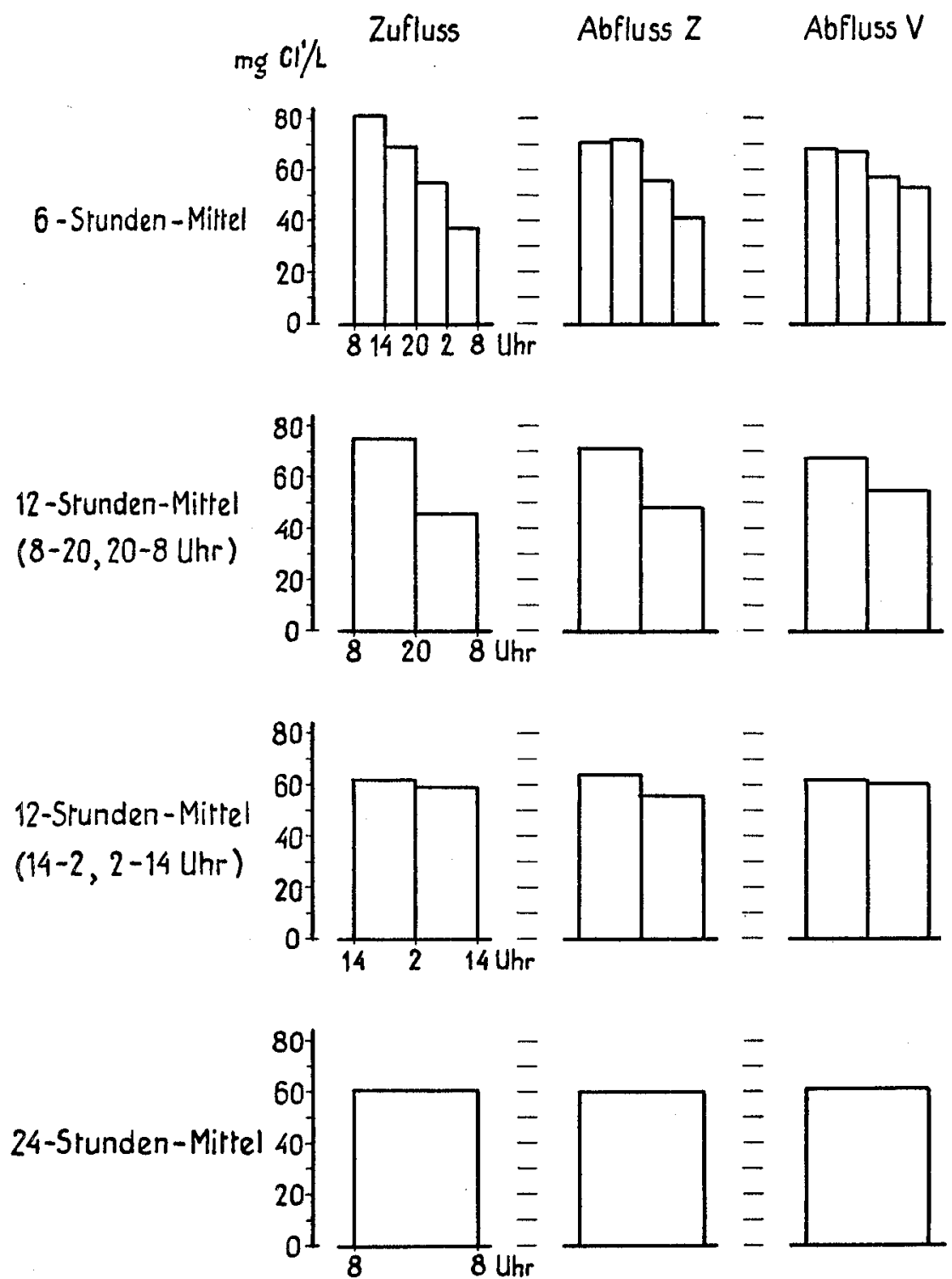

Abb. 2. Chloridgehalt von korrespondierenden Sammelproben zu 6, 12 und 24 Stunden, berechnet aus den Ergebnissen der Einzelproben von Abb. 1. 
in der Anlage erfolgende Gehaltsabnahme schließen, während diejenigen zwischen 20 und 8 Uhr eine Zunahme anzeigen.

d) Grundsätzlich anders verhalten sich 12-Stunden-Sammelproben, welche zwischen 14 und 2 Uhr bzw. 2 und 14 Uhr entnommen werden. Es finden verhältnismäßig geringe Konzentrationsänderungen statt. Solche Proben führen somit zu einem ähnlichen Resultat wie die 24Stunden-Sammelproben.

Die hydraulischen Bedingungen der Anlagen werden noch von weiteren Faktoren mitbestimmt, z. B. von der Menge des Rücklaufschlamms und des Überschußschlamms und anderen, worauf jedoch nicht näher eingegangen werden soll.

Untersuchungen an Tropfkörpern führten zu ähnlichen Ergebnissen.

2. Die Probenahme unter Berücksichtigung der hydraulischen Bedingungen der Anlagen

Wie im vorigen Abschnitt gezeigt worden ist, bewirken die hydraulischen Bedingungen einer Arlage einen Ausgleich der im zufließenden Wasser vorliegenden Konzentrationsschwankungen. Vergleicht man den Gehalt eines an der Reinigung nicht beteiligten Stoffes (Chlorid) des Zuflusses mit demjenigen des Abflusses, so tritt je nach Tageszeit eine Abnahme oder eine Zunahme der Konzentration ein. Daraus muß aber geschlossen werden, daB auch die an der Reinigung beteiligten Stoffe, unabhängig von jeglichem Reinigungsvorgang, dieselben Gehaltsänderungen wie das Chlorid erleiden. Der Endgehalt eines solchen Stoffes richtet sich somit einerseits nach dem Reinheitsgrad des abfließenden Wassers, anderseits nach dem zusätzlich durch die hydraulischen Verhältnisse bedingten Konzentrationsänderungen. Weiter folgt, daß Proben des $\mathrm{Zu}$ - und Abflusses, auch wenn sie zeitlich korrespondieren, in vielen Fällen nicht vergleichbar sind. Auf Grund der erhaltenen Ergebnisse muß die Forderung aufgestellt werden, daß korrespondierende Proben für quantitative Schlüsse hinsichtlich der Reinigungsvorgänge nur dann verwertet werden dürfen, wenn die Chloridwerte identisch sind. Demnach sind zum Beispiel korrespondierende 24-Stunden-Sammelproben als einwandfrei zu bezeichnen. Dagegen sind solche Proben (Sammel- oder Einzelproben) zu verwerfen, die einem Rohwasser-aximum oder -Minimum entsprechen. Als allgemeine Regel gilt: Der Vergleich korrespondierender Proben ist zulässig, wenn die Zusammensetzung der Rohwasserprobe dem durchschnittlichen Tagesgehalt des Rohwassers entspricht. 
Durch diese Regel wird für die Probenahme in Reinigungsanlagen ein neuer Grundsatz aufgestellt, wonach die hydraulischen Verhältnisse der Anlagen berücksichtigt werden müssen. Die Untersuchungen sind in der Weise durchzuführen, daß sich die Entnahme von Proben über einen ganzen Tag erstreckt oder daß für kurzdauernde Prüfungen ein Zeitabschnitt gewählt wird, in welchem niedere und hohe Rohwasserkonzentrationen vorliegen. Allgemein ausgedrückt soll eine Anlage sowohl bei schwacher als auch bei starker Belastung untersucht werden. Andernfalls wird ein Ergebnis erhalten, das für die Arbeitsweise der Anlage während einer längeren Betriebszeit keine Gültigkeit hat.

Für die einzelnen Untersuchungsarten ergibt sich somit:

a) Die sich auf 24 Stunden beziehenden quantitativen Untersuchungen hinsichtlich Reinigungswirkung einer Anlage lassen sich einwandfrei nur auf Grund von 24-Stunden-Sammelproben ermitteln. Der von Zufälligkeiten stark abhängige, unregelmäßige Zufluß läßt es nicht zu, die Werte von Einzelproben oder von während weniger Stunden entnommenen Sammelproben auf den durchschnittlichen Tagesgehalt oder die Gesamttagesmengen zu übertragen.

b) Die Probenahme für die regelmäBig durchzuführenden Betriebskontrollen hat sich nach der oben angegebenen Regel zu richten. Es können nicht beliebig korrespondierende Einzel- oder Sammelproben entnommen werden, sondern es ist darauf zu achten, daß die Rohwasserkonzentration in der Probe ungefähr dem Tagesdurchschnitt des Abwassers entspricht. Es ist also zu vermeiden, ausschließlich bei hohem oder ausschließlich bei niedrigem Gehalt zu untersuchen. Eine allgemein gültige Vorschrift läßt sich nicht angeben, da sich unsere Untersuchungen nur auf Chloridwerte von vorwiegend häuslichen Abwässern stützen und uns der zeitliche Verlauf im Gehalt der übrigen Abwasserstoffe wenig bekannt ist. Unter der Annahme, daß die Gehaltskurve des Chlorids und diejenige der übrigen Verunreinigungen einigermaßen gleichen Verlauf aufweisen, schlagen wir vor, eine Sammelprobe zu entnehmen, die sich etwa über die Zeit von morgens 5 bis 11 Uhr erstreckt. Auch Durchschnittswerte einer Reihe von Einzelproben, welche zu diesen Tageszeiten in regelmäßigen Abständen entnommen werden, führen zu Ergebnissen, bei denen keine den hydraulischen Bedingungen der Anlage zuzuschreibende Fehler vorkommen. Der Vorschlag hat vor allem für die Verhältnisse in unserer Versuchsanlage Werdhölzli Gültigkeit. 
Die Probenahme wurde auf den Vormittag verlegt, um die Analysen gleichen Tags ausführen zu können.

c) Als vereinfachte Methode zur überschlagsmäßigen Ermittlung der sich auf den ganzen Tag beziehenden Werte (mittlerer Tagesgehalt, Tagesmenge) sind die gleichen Arten der Probenahme anwendbar, wie sie für die Betriebskontrollen angegeben wurden. Diese als vorläufiger Hinweis aufzufassende, vereinfachte Methode für Bilanzuntersuchungen kann die während 24 Stunden erfolgende Entnahme von Sammelproben nicht ersetzen. Sie liefert jedoch unter Berücksichtigung der besonderen Verhältnisse der betreffenden Anlage und bei einem Trockenwetterabfluß annähernd richtige Werte.

Da unsere Versuche bei Beschickung der Anlagen mit einer konstanten Wassermenge durchgeführt worden sind, haben die daraus gezogenen Schlüsse nur teilweise Gültigkeit für den allgemeinen Fall der Beschickung mit wechselnder Wassermenge. Die sich über 24 Stunden erstreckenden quantitativen Untersuchungen können in beiden Fällen nach den angegebenen Vorschriften durchgeführt werden. Es wird dann, je nachdem bei der Entnahme der Sammelproben die fließende Wassermenge berücksichtigt wird oder nicht, die Gesamttagesmenge oder der durchschnittliche Gehalt des Tages erhalten. Es ist zu erwarten, da $B$ beim Betrieb mit variabler Wassermenge die hydraulischen Bedingungen der Anlagen sich ähnlich auswirken werden, wie in den beschriebenen Versuchen unter konstanter Wassermenge. Es werden ebenfalls Gehaltsänderungen innerhalb der Anlage festzustellen sein. Man wird in analoger Weise vermeiden müssen, korrespondierende Proben bei einem Rohwassermaximum oder -minimum zu entnehmen. Bestimmte Angaben über das Vorgehen zur einwandfreien Probenahme sind jedoch nicht möglich, solange nicht entsprechende Versuche durchgeführt worden sind.

\section{Beispiele}

Aus unserem Versuchsmaterial greifen wir zwei typische Fälle heraus und vergleichen die Chlorid- und die Kaliumpermanganatwerte. Dadurch soll gezeigt werden, daß auch die mit Kaliumpermanganat oxydierbaren Stoffe ähnliche Gehaltsänderungen erleiden wie die Chloride. Wir schließen dies aus den berechneten Reinigungseffekten, welche je nach der Tageszeit verschiedene Werte annehmen. Unter Berücksichtigung der gleichzeitig festgestellten Chloridgehalte unterscheiden wir zwischen einem «scheinbaren» und einem "wirklichen» Reinigungseffekt. Der erstere, welcher in der Tabelle mit Klammern versehen ist, berechnet sich aus korrespondierenden Proben, die aus den erwähnten Gründen quantitativ nicht verglichen werden dürfen. Der letztere geht aus streng vergleichbaren Proben hervor.

Das Beispiel in Tabelle 4 betrifft vier aufeinanderfolgende 6-StundenSammelproben und die daraus errechneten Werte der 24-Stunden-Sammel- 
Tabelle 4

Chlorid- und Kaliumpermanganatwerte in Sam melproben (Belebtschlammverfahren 9./10.1.47)

\begin{tabular}{|c|c|c|c|c|c|c|}
\hline & & & $\begin{array}{l}\mathrm{Zu}- \\
\text { fluß }\end{array}$ & $\begin{array}{l}\mathrm{Ab}- \\
\text { fluß }\end{array}$ & $\begin{array}{l}\mathrm{Zu}- \\
\mathrm{Abn}\end{array}$ & $\begin{array}{l}\text { oder } \\
\text { ahme }\end{array}$ \\
\hline Kennzahl & Zeit & Nr. & $\mathrm{mg} / 1$ & $\mathrm{mg} / \mathrm{l}$ & $\mathrm{mg} / 1$ & $\begin{array}{c}\% \text { Rei- } \\
\text { nigungs- } \\
\text { effekt }\end{array}$ \\
\hline Chlorid . . . . & $\begin{array}{l}08.00-14.00 \\
14.00-20.00 \\
20.00-02.00 \\
02.00-08.00 \\
08.00-08.00\end{array}$ & $\begin{array}{c}1 \\
2 \\
3 \\
4 \\
\text { Mittel }\end{array}$ & $\begin{array}{r}89 \\
129 \\
80 \\
61 \\
90\end{array}$ & $\begin{array}{r}76 \\
107 \\
85 \\
72 \\
86\end{array}$ & $\begin{array}{l}-13 \\
-\quad 22 \\
+\quad 5 \\
+\quad 11 \\
-\quad 4\end{array}$ & $\begin{array}{l}- \\
- \\
-\end{array}$ \\
\hline $\begin{array}{l}\text { Kaliumpermanganat- } \\
\text { verbrauch . . . }\end{array}$ & $\begin{array}{l}08.00-14.00 \\
14.00-20.00 \\
20.00-02.00 \\
02.00-08.00 \\
08.00-08.00\end{array}$ & $\begin{array}{c}1 \\
2 \\
3 \\
4 \\
\text { Mittel }\end{array}$ & $\begin{array}{l}430 \\
493 \\
329 \\
180 \\
358\end{array}$ & $\begin{array}{l}82 \\
84 \\
76 \\
70 \\
78\end{array}$ & $\begin{array}{l}-348 \\
-409 \\
-253 \\
-110 \\
-280\end{array}$ & $\begin{array}{l}(81) \\
(83) \\
(77) \\
(61) \\
78\end{array}$ \\
\hline
\end{tabular}

Tabelle 5

Chlorid- und Kaliumpermanganatwerte in korrespondierenden Einzelproben

(Belebtschlammverfahren 6.11.46)

\begin{tabular}{|c|c|c|c|c|c|c|}
\hline & & & $\begin{array}{l}\text { Zu- } \\
\text { fluB }\end{array}$ & $\begin{array}{l}A b- \\
\text { fluB }\end{array}$ & \multicolumn{2}{|c|}{$\begin{array}{l}\text { Zu- oder } \\
\text { Abnahme }\end{array}$} \\
\hline Kennzahl & Zeit & Nr. & $\mathrm{mg} / 1$ & $\mathrm{mg} / \mathrm{l}$ & $\mathrm{mg} / \mathrm{l}$ & $\begin{array}{l}\text { o/o Rei- } \\
\text { nigungs- } \\
\text { effekt }\end{array}$ \\
\hline Chlorid . . . . . & $\begin{array}{c}07.00 \\
09.00 \\
-\end{array}$ & $\begin{array}{c}1 \\
2 \\
\text { Mittel }\end{array}$ & $\begin{array}{l}21 \\
64 \\
42\end{array}$ & $\begin{array}{l}36 \\
49 \\
42\end{array}$ & $\begin{array}{r}+15 \\
-15 \\
0\end{array}$ & $\underline{-}$ \\
\hline $\begin{array}{l}\text { Kaliumpermanganat- } \\
\text { verbrauch . . . }\end{array}$ & $\begin{array}{c}07.00 \\
08.00 \\
-\end{array}$ & $\begin{array}{c}1 \\
2 \\
\text { Mittel }\end{array}$ & $\begin{array}{l}177 \\
461 \\
319\end{array}$ & $\begin{array}{l}95 \\
88 \\
91\end{array}$ & $\begin{array}{r}-82 \\
-373 \\
-228\end{array}$ & $\begin{array}{c}(46) \\
(81) \\
71\end{array}$ \\
\hline
\end{tabular}


proben. Der wirkliche Reinigungseffekt beträgt 78\%. Die durchschnittlichen Tageschloridwerte sind praktisch gleich ausgefallen. Dagegen sind bei den 6-Stundenproben die zu erwartenden Änderungen im Chloridgehalt aufgetreten, in den Proben Nr. 1 und 2 im Sinne einer Abnahme, in Nr. 3 und 4 im Sinne einer Zunahme. Dementsprechend ist der scheinbare Reinigungseffekt in den Proben Nr. 1 und 2 gegenüber dem wirklichen zu hoch, bei Nr. 3 und 4 jedoch zu tief ausgefallen. In Tabelle 5 sind die Resultate wiedergegeben, welche mit Einzelproben erhalten wurden. Diese sind ungefähr zur Zeit des Tagesminimums (7 Uhr) und des -maximums $(9 \mathrm{Uhr})$ entnommen worden. Es lassen sich die gleichen, jedoch deutlicher ausgeprägten Befunde wie im vorigen Beispiel feststellen.

\section{Zusammenfassung}

1. Die hydraulischen Bedingungen einiger mit konstanter Wassermenge beschickten Abwasserreinigungsanlagen sind mit Hilfe systematischer Chloridgehaltsbestimmungen untersucht worden. Dabei wurde festgestellt, daß das Abwasser beim Durchfließen einer Anlage Konzentrationsänderungen erleidet, welche sich abwechslungsweise im Sinne von Zunahmen und von Abnahmen äußern.

2. Es konnte gezeigt werden, daß der bisher bei der Untersuchung von Reinigungsanlagen eingehaltene Grundsatz, wonach korrespondierende Proben zu entnehmen sind, in vielen Fällen nicht genügt und zu falschen Ergebnissen führt.

3. Es wird vorgeschlagen, bei der Prüfung von Reinigungssystemen Probenahmemethoden anzuwenden, bei welchen auf die hydraulischen Verhältnisse der Anlage Rücksicht genommen ist. Die dabei einzuhaltenden Regeln werden näher umschrieben.

4. Die Einhaltung des vorgeschlagenen neuen Grundsatzes ist besonders in denjenigen Fällen notwendig, in welchen eine Anlage auf Grund von Sammelproben während weniger Stunden oder von Einzelproben untersucht wird (Betriebskontrollen und andere kurzdauernde Versuche). Dagegen wirken sich die hydraulischen Bedingungen nicht mehr aus, sofern 24-Stunden-Sammelproben entnommen werden.

5. Es wurde geprüft, wie sich die Reinigungsvorgänge in einer Anlage quantitativ erfassen lassen. Bei der Vielseitigkeit der gegebenen Bedingungen konnten die Vorschläge zur Lösung der gestellten Fragen nicht durchwegs einfach ausfallen. Doch stehen nun Methoden zur Verfügung, welche zunächst bei konstanter Beschickung den verschiedenen Umständen besser Rechnung tragen und zur quantitativen Abklärung der sich in Reinigungsanlagen ereignenden Vorgänge beitragen werden. 


\section{Literatur}

(1) IMHOFF, K. Ges.-Ing. 68.169 (1947).

(2) BACH, H. Ges.-Ing. 52. 65 (1929).

(3) MEINCK, F. Die Stadtentwässerung in Deutschland (Brix, Imhoff, Weldert). Jena 1934.

(4) BABBITT, H. E. Sewerage and Sewage Treatment, New York und London 1947.

(5) THEROUX, F. R., ELDRIDGE, E. F., und MALLMANN, W. Laboratory Manual for Analysis of Water and Sewage, New York und London 1936.

(6) AM. PUBLIC HEALTH ASSOCIATION. Standard Methods for the Examination of Water and Sewage, New York 1946.

(7) TILLMANS, J. Die chemische Untersuchung von Wasser und Abwasser, Halle 1932.

(8) Einheitsverfahren der Wasseruntersuchung, Verlag Chemie, Berlin 1940.

(9) KROPF, A, Straße und Verkehr 52. 72 (1940).

(10) ZIGERLI, P. Ges.-Ing. 60.499 (1937).

(11) KESSENER, H. Ref. in Ges.-Ing. 54. 93 (1931). 